\title{
Modified coupled map car-following model by considering the effect of multiple preceding vehicles ZHANG Kun ${ }^{1, a}$, ZHOU Tong ${ }^{1 *}$ \\ ${ }^{1}$ College of Information Engineering, Chongqing Institute of Engineering, Chongqing, 402260, China \\ a 2530870471@qq.com
}

Keywords: traffic flow; traffic congestion;CM car-following model; effect of multiple preceding vehicles

Abstract: Based on Konishi et al.'s reaserch achievement, a modified coupled map (CM) car-following model referring to the OV function of multiple preceding vehicles is proposed to study the effect of multiple preceding vehicles on the propagation characteristic of traffic flow wave. According to the theory of linear systems, the stability conditions of traffic flow when the speed of the leading car changed are obtained and the research results are compared with previous studies. Numerical simulation shows that the modified CM model can improve traffic flow stability, and the traffic jam can be more effectively suppressed is better than the traditional CM model.

\section{Introduction}

With the rapid economic development, traffic congestion is being concerned. In order to explore the internal mechanism of traffic jams, many scholars proposed lots of traffic flow models. In 1995, Bando et al. [1] proposed to optimize speed (OV) model which can describe the actual traffic phenomena. Coupled map (CM) - following model [2] is a discrete version of the OV model, which can reveal the movement rule of traffic flow, and also research congestion suppression problem. In 1999, based on the CM-following model, Konishi et al. [3] proposed congestion delayed feedback control method to achieve control of the running vehicles, the simulation results demonstrate its effectiveness. In 2006, Zhao et al. [4] proposed a vehicle's speed difference information method on the basic of Konishi et al.'s method, which has more simple form, and numerical simulations show that suppression performance of congestion was better than the literature [3]. In 2007, Han et al. [5] introduced the traffic guidance information into CM model, the considered car can more timely and accurate obtain its ahead traffic conditions, and take timely measures to control the speed for stability of traffic jam and suppression traffic congestion. In 2009, Shen et al [6] studied the influence of the feedback signal about the relative speed information between the considered car and the nearest dual front vehicle affect the stability of traffic flow, simulation results show that congestion suppression strategy can significantly improve congestion condition.

However, the congestion suppression studies mentioned above based on CM car-following model just consider the vehicle's running state information in feedback control method, which donot improve the CM car-following model. The results show that modified $\mathrm{CM}$ car-following model can enhance the stability of traffic flow, and the multiple preceding vehicles' position information also improve stability of traffic flow[7]. Therefore, based on Intelligent Transportation Systems (ITS) provides guidance information, a new CM-following model considering multiple vehicles' headway information is proposed. The simulation results are compared with the previous results. 


\section{Modified CM car-following model}

In this paper, we first give the modified CM following model. The motion equations of the preceding car is described as follows:

$$
x_{0}(n+1)=v_{0} T+x_{0}(n) .
$$

where $x_{0}(n)$ is the position of the leading vehicle at time $t=n T, x_{0}(0)$ is the leading vehicle's initial position and $v_{0}$ is its velocity which is a constant, $T$ is the sampling interval.

The motion equations of following vehicle $i$ is described as

$$
x_{i}(n+1)=v_{i}(n) T+x_{i}(n) .
$$

where $i=1,2, \ldots, N, x_{i}(n)$ and $v_{i}(n)$ are the position and speed of the $i$-th vehicle at time $t$ $=n T$ respectively, $\mathrm{N}$ is the total number of vehicles.

The headway $y_{i}(n)$ between the following vehicle $i$ and its ahead vehicle $i-1$ is described as: $y_{i}(n)=x_{i-1}(n)-x_{i}(n)$. The speeds of the following vehicles are governed by

$$
v_{i}(n+1)=\alpha_{i}\left[V_{i}^{O P}\left(\sum_{m=1}^{s} p_{m} y_{i-m+1}(n)\right)-v_{i}(n)\right] T+v_{i}(n) .
$$

where $a_{i}$ is the sensitivity of the $i$-th vehicle driver, $\sum_{m=1}^{s} p_{m} y_{i-m+1}(n)$ is the OV function, $p_{m}$ is weight parameter, which depends on the headway distance

$$
y_{i-m+1}(n)=x_{i-m}(n)-x_{i-m+1}(n) .
$$

Where $m=1,2,3, \ldots, s$ is the number of vehicles which can provide guidance information, The $\mathrm{OV}$ function is given by

$$
V_{i}^{O P}\left(\sum_{m=1}^{s} p_{m} y_{i-m+1}(n)\right)=\frac{v_{i}^{\max }}{2}\left[1+\bar{H}_{s a t}\left(2 \frac{\sum_{m=1}^{s} p_{m} y_{i-m+1}(n)-\eta_{i}}{\zeta_{i}}\right)\right] .
$$

where the parameters are consistent with that of the literature [3].

\section{Stability analysis}

The initial condition is consistent with that of the literature [3]. The dynamic behaviour of the $i$-th vehicle can be given as

$$
\begin{aligned}
& v_{i}(n+1)=\alpha_{i}\left[V_{i}^{O P}\left(\sum_{m=1}^{s} p_{m} y_{i-m+1}(n)\right)-v_{i}(n)\right] T+v_{i}(n), \\
& y_{i}(n+1)=v_{0}(n) T-v_{i}(n) T+y_{i}(n) .
\end{aligned}
$$

The steady state of vehicle system (10) is

$$
\left[v_{i}^{*}, y_{i}^{*}\right]^{T}=\left[v_{0}, \stackrel{\circ}{\stackrel{s}{a}}\left(1-\frac{1}{p_{m}}\right)^{i-m}\left(y_{1}-\frac{v_{0}}{r_{i}}+\frac{z_{i}}{2}-h_{i}\right)+\frac{v_{0}}{r_{i}}-\frac{z_{i}}{2}+h_{i}\right]^{T},
$$

where $r_{i}=v_{i}^{\max } / \zeta_{i}$. In order to analyze the stability of the system (6), a perturbation model 
near steady state of the system(6) is given by using perturbation method.

$$
\begin{aligned}
& \delta v_{i}(n+1)=\left(1-\alpha_{i} T\right) \delta v_{i}(n)+p_{1} \alpha_{i} r_{i} T \delta y_{i}(n), \\
& \delta y_{i}(n+1)=-T \delta v_{i}(n)+\delta y_{i}(n) .
\end{aligned}
$$

The perturbation model(8) is changed into a state space form as follows

$$
\left[\begin{array}{l}
\delta v_{i}(n+1) \\
\delta y_{i}(n+1)
\end{array}\right]=\left[\begin{array}{cc}
1-\alpha_{i} T & p_{1} \alpha_{i} r_{i} T \\
-T & 1
\end{array}\right]\left[\begin{array}{l}
\delta v_{i}(n) \\
\delta y_{i}(n)
\end{array}\right]
$$

After the ith vehicle is disturbed, it can eliminate the influence of the disturbance, and restore the steady state. The process can be seen as the stability problem of the disturbed system(6) .

The transfer function $G$ from $d v_{i-1}(n)$ to $d v_{i}(n)$ is described by

$$
G=\left[\begin{array}{cc}
1-\alpha_{i} T & p_{1} \alpha_{i} r_{i} T \\
-T & 1
\end{array}\right]
$$

Also according to the control theory of the literature [5], the ranges of the parameters of traffic system are given as

$$
0<r_{i}<\frac{1}{p_{1} T}, \quad 0<\alpha_{i}<\frac{4}{\left(2-p_{1} r_{i} T\right) T} .
$$

This condition is a necessary and sufficient condition to keep system (9) to be stable.

\section{simulations}

Our simulations are based on the CM car-following model under open boundary conditions. The CM car-following model parameters are consistent with that of the literature [3]. In view that the influence of the preceding vehicles on the current vehicle reduces gradually as the distance between them increases, thus $p_{m}$ decreases monotonically with the increasing $m$, that is, $p_{m+1} / p_{m}<1$.In a way similar to that presented by Ge et al., assume that $p_{m}=\frac{2}{3^{m}}(m=1,2 \ldots, s-1), p_{s}=\frac{1}{3^{s-1}}$. Consider a situation where the leading vehicle stops suddenly for a four short time[5]: $v_{0}(n)=0, \quad n T=100-102 \mathrm{~s}, \quad 120-122 \mathrm{~s}$, 140-142s, 160-162s. The external disturbance is used to examine the stability performance of our model on traffic flow.

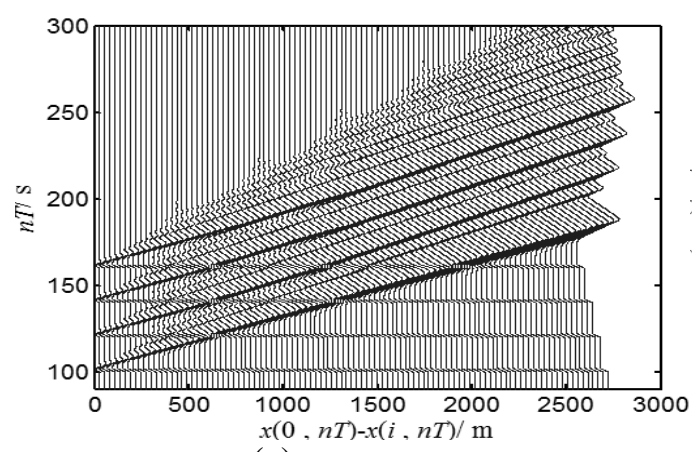

(a)

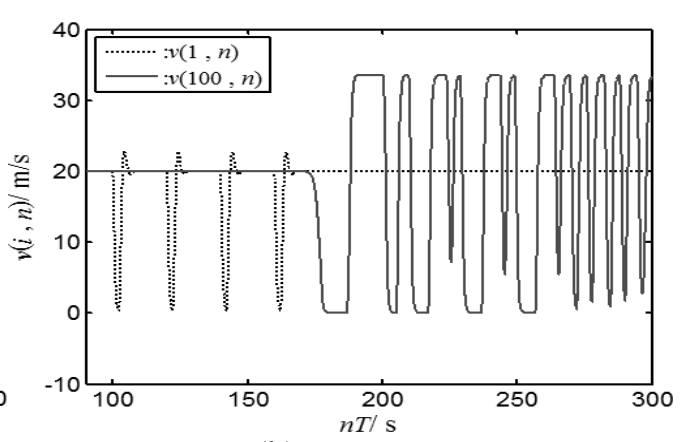

(b)

Fig. 1. Numerical simulations for CM car following model. 

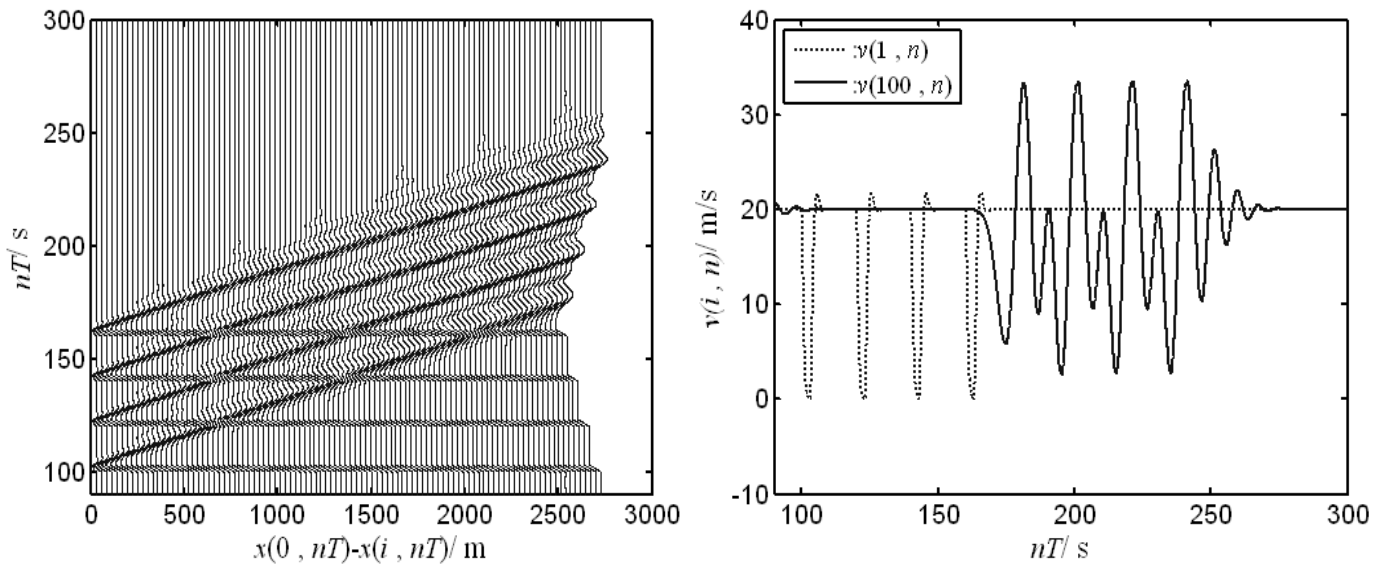

Fig. 2. Numerical simulations for Ge et al.'s [9]car following model.

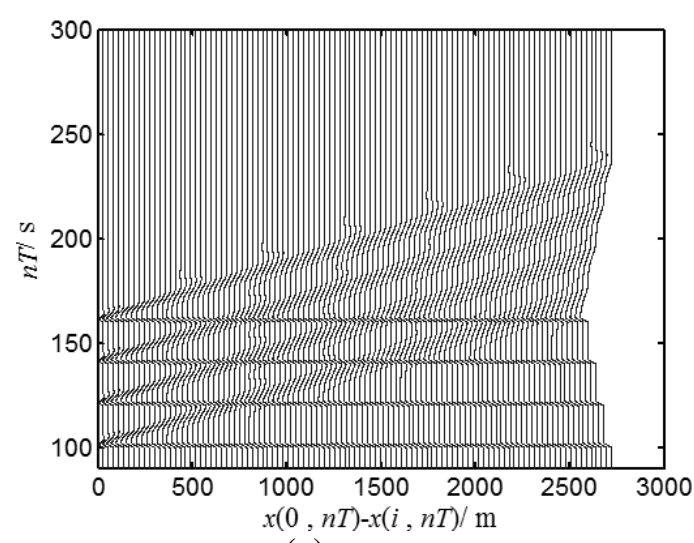

(a)

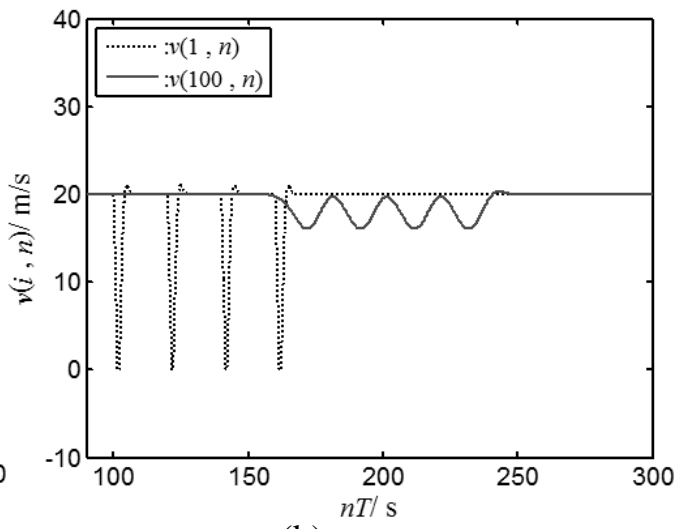

(b)

Fig. 3. Numerical simulations for our model.

Figures 1(a), 2(a) and 3(a) show the space-time plot of the traffic flow in the CM car-following model, the model of Ge et al.[9] and our model. It can be found that the stop disturbance propagates backwards in Figures 1(a), 2(a) and 3(a). From Figure 3(a), we find that there is no oscillating behavior and traffic jam, and the degree of traffic congestion is the smallest, thus the traffic system can back to no congestion state in a shortest time, compared with those in Figures 1(a) and 2(a). Figures 1(b), 2(b) and 3(b) show the temporal velocity behavior of the first, 25th, and 50th vehicles according to Figures 1(a), 2(a) and 3(a) respectively. In Figure 1(b), it is found that traffic jam occurs without any control signal, and the transient behavior of each vehicle propagates backward with the increase of its amplitude. From Figures 2(b) and 3(b), it is obvious that the vehicles are moving smoothly as the headway information is introduced in the CM car following model. However, the amplitude of speed fluctuation in our model is smaller than that in the model of Ge et al. These simulation results show that the stability performance of our model on traffic flow is better than those of the two previous models.

\section{Conclusions}

In this paper, a new $\mathrm{CM}$ car following model is proposed by considering multiple preceding car's headways. The stability of the model is investigated, which can better reflect the characteristics of the traffic flow in the modern traffic flow, and can effectively suppress the traffic congestion caused by the preceding car's disturbance, and improve the stability of 
the traffic flow.

\section{Acknowledgements}

This work was financially supported by the China Postdoctoral Science Foundation Funded Project (Grant No. 2015M572450), Scientific and Technological Research Program of Chongqing Municipal Education Commission(Grant No.KJ1503301).

\section{References}

[1] M. Bando, K. Hasebe, A. Nakayama, Dynamical model of traffic congestion and numerical simulation, Phys. Rev. E, 51(1995) 1035-1042.

[2] S. Yukawa, M.Kikuchi, Density fluctuations in traffic flow, J. Phys. Soc. Jpn., 65(1996) 916-919.

[3] K. Konishi, H. Kokame, K. Hirata, Coupled map car-following model and its delayed-feedback control, Phys. Rev. E, 60(1999) 4000-4007.

[4] X.M. Zhao, Z.Y. Gao, A control method for congested traffic induced by bottlenecks in the coupled map car-following model, Physical A, 366(2006) 513-522.

[5] X.L. Han, C.Y. Jiang, H. X. Ge, S.Q. Dai, A modified coupled map car-following model based on application of intelligent transportation system and control of traffic congestion, Acta. Phys. Sin 56(2007) 4383-4392.

[6] F.Y. Shen, H.X. Ge, H. Zhang, A control method for congested traffic in the coupled map car-following model, Chin. Phys. B, 18(2009) 4208-4216.

[7] R. J. Cheng, X. L. Han, S.M. Lo, H. X. Ge, A control method applied to mixed traffic flow for the coupled-map car-following model, Chin. Phys. B,23(2014):030507. 